\title{
Übersicht des Inhalts.
}

I. Einleitung .......... I- 27

Verhältnis der alındischen Kunst zur allgemeinen Kunstgeschichte. Abhängigkeit ohne Rückwirkung. Das religionsgeschichtlıche Interesse vorberrschend If. Chronologısche Stellung, rückläufige Entwicklung 2. Die Denkmäler 3.

Hỉ lfsmittel zur Érklärung der Skulpturen 3 f. Versuch die Haupttypen der dargestellten Wesen und der beliebtesten Kompositionen festzustellen. Ikonographische Litteratur der nördlichen Schule des Buddhismus 4.

Skızze der altındischen Kulturgeschichte $4 \mathrm{ff}$ Uıe Arya im Pandschâb (Nordwestl. Indien) 5. Die Götter der Vedaperode 5. Cakra in Veda. Sakka in den Pâlltexten 6. Kein Kultbild in der nordischen Terıode 6.

Technische Kenntnisse der alten Ârya nach der vedischen Litteratur $6 \mathrm{ff}$ Das Speichenrad ein gepriesenes Meisterwerk 6 Steınbauten. Wehrmauern und Stüpen, Goldschmuck 6-7.

Die îrya im Gangesiande 7 ff. Das 5. Jahrhundert v. Chr. Inder, Perser, Iellenen 7. Machtstellung des persischen Reiches unter den Achämeniden 8. Buddha's Lehre die erste Weltreligion 8. Beziehungen der Achämeniden zu Indien. Hindhù und Gandârì als Linterthanen des Perserkönigs 8f. Die Staaten von Magadha und Koçala of.

Religiöser Zug des indischen Kulturlebens ıoff. Die Philosophie ro. Die Seclenwanderung I0. Lehre von der Weltseele I I. Brahman und Âtman II. I Je Befreiung II.

Der Buddha 12 ff. Gautama's Geburtsort, seine Jugend 12. Die vier Erscheinungen 13. Gautama verlässt seinen Palast und wird Asket I3. Der "Erleuchtete" 13. Buddha's Tod 14. Das erste und zweite Konzil I4. Die Maurya's auf dem Throne des Reiches Magadha (Prasioi) 15. Tschandragupta, Afoka, seine Bauten und seine Edikte 15. Persischer Einflufs in Indien 15-16. 
Açoka's Bauten, Stil derselben 16. Die persische Säule 17. Orientalisierende Tiere 17. Flagelwesen 18. Stilisierte Pflanzen 18 . Daneben die malerisch behandelte einheimische Flora, Lotusmuster 19.

Zweck der Denkmaller 20ff. Stambha's, Tschaitya's, Vihâra's, Stûpa's, Railings mit Thoren 20-2r.

Zwei Gruppen: persisch-indischer und graecobuddhistischer Stil 21 ff. Barâhat 24. Sàñtschí 24. Amarâvatí 26. Einflufs der Gandbàra-Schule: Adschaṇtà 26. Alte Malschule 27.

II. Der persisch-Indische stil ...........

Altindische Kunstubung $28 \mathrm{ff}$. Holzschnitzstil in Stein Ubertragen. Thore und Thronsessel 29. Dekorativer Charakter der indischen Kunst 30. Gold sc h miede kunst 31. Einfluis des Schmuckes auf die Darstellung des menschlichen Körpers ein Hindernis fur die Plastik 32.

Stoffe der Darstellungen: Die einzelnen Figuren $33 \mathrm{ff}$. Der Mensch 33. Mythische und wirkliche Fremdvölker, der Hindâtypus 34. Zwerge 35. Bekleidung und Schmuck $36 \mathrm{ff}$. Betonung der Huftenpartie durch das Gewand bedingt 37 .

Die Gotter und Halbgötter $38 \mathrm{ff}$. Çakra, der Donnergott und die tubrigen Gotter 39. Der Donnerkeil 39. Die Gøttin Sirf (Çrî) 40. Niedrige Gottheiten auf ihren Attributen stebend 4I. Mischwesen $42 \mathrm{ff}$. Der Nâga (Schlangendămon), zwei Typen: einer nach Art der Uräos-Schlange: ganz menschlich mit Schlange im Nacken, einer mit menschlichem Oberkorper, tierischem Unterkbrrper 43 ff. Matsyanârís 44. Flügelwesen 44. Devatâ's, Kinnara's 45. Blütterschurz oder Vogelleib 47.

Garuda 47 ff. Garudmant 47. Papageientypus 48. Gryps, Cherub 49f. Die goldhutenden Greife 49. Tibetische Hunde, der koreanische „Löwe", Thien-kou: Ten-gu 51.

Pferdek bpfige Todesdamonen 50.

Kentauren: Tiryagyoni 5 I.

Indische Erklurung der orientalisierenden Tiere $\mathbf{5 2} \mathrm{ff}$. Mărchenbildung aus fremden Kunstformen 52. Thronlehnen und Pfeilerdekorationen 53. Sabbadàthadschâtaka 54 f. Einfiuss der Kunst anf die buddhistische Litteratur 55. Das Motiv des "unter die Fufse Tretens" 56. Griechisches unter den orientalisierenden Tieren: Makara (Delphin), Hippokampen, Kentauren, Pygmäen 57.

Die Komposition 57ff. Erzahlende Reliefs: Prozessionen zu beiligen Orten: Grtter, Menschen, Tiere dabei beteiligt 58. Formelhafte Abkurzungen und Wiederholungen 58. Die formelhaflen Wiederholungen in den heiligen Texten 58. Genrescenen in den Reliefs. Naturschilderungen, welche die Komposition begleiten 59. Stadtbilder mit reich belebten Terrassen: Terrassen der Gotterhimmel 60. 
Seite

Scenen aus Buddba's Leben ohne Buddhadarstellung en 6r ff. Die Kâçyapa-Legende am östlichen Thore von Sâñtschî 6r. Das Feuer- und Wasserwunder von Uruvilvâ $63 \mathrm{ff}$. Folgescenen a uf einer Platte 65 . Stark betonte Nebensachen: breite Naturschilderung 65. Dieselbe Erscheinung in der Litteratur 67. Buddha nur durch Symbole dargestellt 68. Parallele Erscheinung in der fruhchristlichen Kınst 69.

Mittel, die Komposition verständlich zu machen $70 \mathrm{ff}$. Der $\mathrm{Zusammenhang} \mathrm{einer} \mathrm{Reihe} \mathrm{von} \mathrm{Reliefplatten} \mathrm{auf}$ einem Monumente erklärt die Einzelndarstellungen 7off. Dekorative Elemente dienen zur Bestimmung der Darstellungen $\mathbf{7 2 .}$

Architrave des östlichen Thores von Sâñtschî: das Wappen von Ceylon, das der Mauryakönige 71. Der Fusstapfen Buddha's 73.

III. Die Gandhârâ -Skulpturen (sogen. Graeco-buddhistische Skulpturen) . . . . . . . . . . .

Politische Geschichte 74. Das griechisch-bakrische Reich, Menandros 75 .

Antiker Einflufs a uf die Länder des Ostens 75. Indien und Ostasien $76 \mathrm{f}$. Missionen des Buddhismus 76. Die Yue-tschi oder Indoskythen 77. Konzil von Dschâlandhra 77. Nördliche Schule des Buddhismus 77. Guptadynastie in Indien 78. Manichäismus, der Paraklet 79. Gandarioi und Indoi 79.

Einflüsse der entwickelten griechischen Kunst auf die buddhistische 80 . Indohellenische und Gandhâra. Schule 8o. Chronologische Stellung der GandhâraSchule 8t. Inncre Ähnlichkeit mit der italischen Kunst 8r. Die Gandhâra-Schule repräsentiert einen Ableger der antiken Kunst, aber die Stoffe sind rein indisch; in der nördlichen leben die Formen fort $8 \mathrm{t}$.

Die Typen $82 \mathrm{ff}$. Der Buddhatypus, Gautana als Buddha $82 \mathrm{ff}$. Der Nimbus 83 . Ty pen der Götter $84 \mathrm{ff}$. Brahmâ und Çakra 84 . Der sogenannte Devadatta $85 \mathrm{ff}$. Verschiedene Typen des Donnerkeilträgers 86f. Bärtiger und unbärtiger Kopf des Gottes 88 . V a d s chrapạni und andere neue Namen für ältere Typen 90. Zwei Donnerkeilträger auf einem Relief $9 \mathbf{r}$. Vadschrapâni ein Yaksha und ein Bodhisatva 92. Màra mit Pfeil und Bogen 92. Lokalgottheit: Gottheit des Palastthores 93. Mâra's Heer 94. Die Göttinn der Erde: antiker Gê-Ty pus, Einfluss der Kunst auf die spätere Litteratur 96. Yaksha's das Pferd des Bodhisatvas haltend 99. Unbestimmbare Göttinnen: die Typen der japanischen Ben-ten roo, indischen Sarasvatî ror. Nâga's rc2. Nâgî und Garuda I03: der Ganymedes des Leochares 103. Deva dâsî: Nâtschmädchen unter dem Baume stehend I04. Darstellung der Geburt Buddha's I03.

Der Todesbote ro6. Die Brâhmạ̣a's 107. Könige, Frauen, Yavanânî's 108-109. 
Kunstlerische Bedeutung der Gandhâra-Schule 109. Handwerksmăfsige Reproduktion eitier Reihe fertiger Typen 109. Alte Musterkompositionen: Fortlehen derselben in der nöndlichen Schule 110-int.

Die Komposition It If. Antikes Schema, Kepliken z. B. die Gebuut, đie Flucht aus dem Hause, Predigtscenen, der Tod (Nirvàna) 111 ; in voller und abgekurzter Form, nur in Reihen erklfibar. Darstellung des Nirvâna in Gàndtàra, Trbet, China und Japan 112 bis 116. Vorlage: antike Sarcophagreliefs 116. Kombinierung mehrerer Masterkompositionen auf einer Platte (das arfindische Motiv der Folgescenen bricht wieder durch) mit gleichgrofsen Figuren (ältere Art), mit verschieden grofser Figuren (jungere Art) 117. Die Kàçyapa-Legende als kombinirte Komposition 118, als einzelne Komposition 119, in ganz abgekurzter Form 120. Andere kombinierte Platten 121. Das Verlassen des Hauses 121.

Stelenkomposition: Mittelfeld ein Buddha oder Bodhisatva mit zwei Begleitern 122. Moderne Gemälde im Stil solcher Stelen 123 .

Die dekorativen Elemente 124ff. Kauernde Garuda's 125. Atlanten 126. Pfeilerfiguren 126. Der Tributtrăger 126.

WeIthtuter (lokapàla's) und Kunstporträts 127. Kubera und Virudduaka $128-129$. Ho-shang der Vertreter des Mahâyàna 129.

Grofse und kleine (dienerde) Figaren 130 Paignia 130. Guirlandenträger 130.

Alte Symbole: Rad und Dreizack I31.

Architektoniscbe Elemente: robınische Pfeiler neben irdopersischen Säulen 132.

Einfinfs der Gandhâraschule auf die indische Kunst 133.

Antikes in den Skulpturen von Amaravati 133-134.

IV. Darsțellang vou Bredha and Bodhisatva. . . . . . 135-183

Tschakravartf́ $\mathbf{3} 35$ f. Die sieben Juwrlen 136. Übcrnaturlicbre Attribute der Buddhagestalt 137. Die grossen und kleinen Schönheitszeichen' $138 \mathrm{f}$. Schematisierung der Buddhafigur und philosophischische Untersuchung des Buddhal,egriffs 140f. Buddha's Bild geschaffen durch Anlehnung an das Apollo-Ideal 140f. Zwei Richtungen, eine idealistische und realistische mit indischen Abarten 143. Indo-baktrische Malschule in China, Korea, Japan 145. Buddha mit Schnurrhart 146. Gewa nobehandlung 146. Die griechische Faltenlegung traditioncll gut crhalten 147. Das Sandelholzbild des Königs Udayana 148. Legendarische Erklärung der fremden Formen 149. Weiterleben des Gandbârastils der Buddhafigur 150 . Typen $m t$ unbedeckter rechter Schulter scbon in Gandhâra 151. Grkronte Buddhafiguren in Hinterindien 153 Die verschiedenen Stellungen der Buddhafigur 155. Die Stellung der Hănde; die Mudrà's 157 . 
Die fruheren Buddba's und der kommende $158 \mathrm{ff}$.

Maitrêy a 158. Die Bodbisatva's in kgl. Tracht und Schmuck dargestellt 159. Wie weit sind die Mudra's (Handstellungen) in Gandhàra für die Namen bestimmend 160. Moderne Darstellung des Maitrêya 16r $\mathrm{f}$. Maitrêya mit Gefals in Gandhâra 163. Buddhafiguren mit Dharmatschakramudrâ 165 stellen, wenn dem Gautama gegenuber gestellt, den Maitrêya dar 166. Bestimmte Mudrâs, ursprunglich nur Phasen im Leben eines Buddha, werden später für bestimmte Buddha's typisch. Kâcyapa 166. Maitrêya's dominierende Stellung in der Mahâyânascbule 167 .

Bodhisatvafiguren mit Lotosbluten 168 . Padmapâni und die Dhyânibuddha's 169.

Iranische Elemente 170: die Fravashi's der Iranier 170. Wiederholung des Buddha- und Bodhisatvatypus i 7 I ff. Scbematische Wiederholung und Masconproduktion 172. Aufhebung des iadividuelken Charakters 172 Kolosse 173.

Der Lotusthron 173.

Die letzten ausgeprägten Gestalten: Mañdschugrif und Padmapani 174. Meditiation als Ideal des Kopfes 176. Der Allerbermer 177. Dauerbaftigkeit des antiken Typus 177.

Annäherung an die Personifikation 177.

Gruppen von Figuren 178. Triaden, Pentaden 178. Vielgliedrigkeit 178 .

Reaktion dagegen 178. Das Lamen-Porträt in Tibet, China, Japan 179. Die Karikatur in Japan 179. Die Karikatur in Japan benutzt gelegentlich auch alte Kompositionen r81.

Keine Kunstlernamen 182. Die Kunstler nach der Öberlieferung: Yaksha's und Nàga's 183

Das netionalindische Element wirkt aufiosend durch die stete Wiederholung ins Mafslose und durch die Systematisierung im einzelnen 183.

\section{Anhang 1.}

Noten ....................... 185-194

Anhang II.

Reliefs des đ6tlicben Thores von Sâñtschf. . . . . . . 195-197

Origiealskulpturen aus Buddhagayâ. . . . . . . . . 197

\section{Anlang III.}

Originalskulpturen aus Gandhâra. . . . . . . . 198-200

Verzeichnis der Gipssbgusse. . . . . . . . . . . . . 201

Terrakotten aus Turkistán. . . . . . . . . . . 201

Index . . . . . . . . . . . . $202 \mathrm{ff}$ 